“(C) 2018 IEEE. Personal use of this material is permitted. Permission from IEEE must be obtained for all other uses, in any current or future media, including reprinting/republishing this material for advertising or promotional purposes, creating new collective works, for resale or redistribution to servers or lists, or reuse of any copyrighted component of this work in other works." 


\title{
Bandwidth-Enhanced, Compact, Near-Field Resonant Parasitic Filtennas with Sharp Out-of-Band Suppression
}

\author{
Ming-Chun Tang, Senior Member, IEEE, Ying Chen, Student Member, IEEE, Ting Shi, Student Member, IEEE, \\ and Richard W. Ziolkowski, Fellow, IEEE
}

\begin{abstract}
The designs, simulations, and measurements of a class of compact, bandwidth-enhanced filtennas are reported. Our design strategy is illustrated by separately designing a monopole and a band-pass filter to operate primarily in their respective fundamental modes. By combining these elements and manipulating the mutual coupling between them, an enhanced impedance bandwidth filtenna is realized. This strategy is applied to augment metamaterial-inspired NFRP antennas with filters. Simulations of these filtenna systems demonstrate that one can maintain stable radiation performance characteristics no matter how one arranges their component configurations, i.e., their relative positions and orientations. A selected filtenna design prototype was fabricated and tested. The good agreement between the simulated and measured results validates these design principles.
\end{abstract}

Index Terms-Antennas, electrically small antennas, filtennas, impedance bandwidth, near-field resonant parasitic elements

\section{INTRODUCTION}

A s a hybrid component in RF front-end systems, filtennas $\mathrm{A}_{\text {possess }}$ the distinctive advantage of simultaneously combining filtering and radiating performance characteristics. Thus, they not only save space and costs, but also reduce transmission losses that occur in traditional arrangements in which the filter and antenna are cascaded as separate units. As a consequence, filtennas have captured much research attention over the past decade [1]-[6].

In general, the reported filtennas can be classified into three main categories. One class, band-stop filtennas, focuses on introducing band-notch filters into ultra-wideband (UWB) / wideband antennas using a variety of high-Q band-stop

Manuscript received on May 1, 2018, and revised on June 06, 2018...

This work was supported in part by the National Natural Science Foundation of China contract number 61471072, in part by the Funding of the Innovative Leading Talents in Science and Technology of Chongqing contract number CSTCCXLJRC201705, in part by the Opening Subject of State Key Laboratory of Millimeter Waves under Contract K201732, in part by Funding of the Young Backbone Teachers in Colleges and Universities of Chongqing contract number 0307001104102, and in part by the Australian Research Council grant number DP160102219.

M.-C. Tang, Y. Chen, and T. Shi are with are with the Key Laboratory of Dependable Service Computing in Cyber Physical Society Ministry of Education, College of Communication Engineering, Chongqing University, and also with the State Key Laboratory of Millimeter Waves, Nanjing 210096, China (tangmingchun@cqu.edu.cn);

R. W. Ziolkowski is with the University of Technology Sydney, Global Big Data Technologies Centre, Ultimo NSW 2007, Australia, and the Department of Electrical and Computer Engineering, University of Arizona, Tucson, AZ 85721, USA (E-mail: Richard.Ziolkowski@uts.edu.au). structures [1], [2]. The second class, band-pass filtennas, are obtained by embedding band-pass filter structures into various types of basic antennas [3], [4]. The third class, multi-resonator-cascaded filtennas, is obtained by altering the coupled-resonators in the last stages of the filters to act as the radiating elements [5], [6]. For the first two categories, the impedance bandwidths of filtennas are confined to be within the antenna's original impedance bandwidth, and in theory, cannot exceed this range of frequencies [1]-[4]. For the third category, the multi-resonator-cascaded design strategy makes their electrical sizes inescapably not small [5], [6]. Therefore, it is highly desirable for many practical applications to achieve a filtenna that not only possesses an impedance bandwidth wider than that of its antenna components alone, but is also compact.

In this letter, a filtenna with both compact structure and enhanced bandwidth is developed with a novel design strategy. It attains an impedance bandwidth that is more than a 3 times improvement in comparison to its component near-field resonant parasitic (NFRP) monopole antenna alone. A set of compact, NFRP filtennas are simulated and analyzed to verify the reliability of the filtenna. Note that all of the reported designs employ a Rogers 4350B substrate with relative permittivity $\varepsilon_{r}=3.48$, relative permeability $\mu_{r}=1.0$, and dielectric loss tangent $\tan \sigma=0.0037$. All of the numerical simulations and optimizations were performed using the frequency domain finite element-based ANSYS/ANSOFT high frequency structure simulator (HFSS), version 13.0.

\section{Design of A Monopole-BASEd Filtenna}

Fig. 1 depicts a simple band-pass filtenna design consisting of a traditional quarter-wavelength cylindrical monopole and a microstrip rectangular capacitively loaded loop (CLL) -based band-pass filter that operate near $1.25 \mathrm{GHz}$ [7]. The monopole (with radius of $2.5 \mathrm{~mm}$ and height of $57 \mathrm{~mm}$ ) is oriented vertically to and above a circular substrate disk with a $0.762 \mathrm{~mm}$ thickness and a $75 \mathrm{~mm}$ radius. The CLL-based filter is etched on the top side of the substrate; it consists of a CLL element and two L-shaped microstrip coupling segments. The bottom side of the substrate acts as the ground; the outer wall of the $50 \Omega$ coax feed line is connected to it. The two L-shaped microstrip coupling segments, whose characteristic impedance is $50 \Omega$, connect the monopole and the center conductor of the coax feed line to the L-shaped coupling segments of the filter.

The filtenna illustrated in Fig. 1 is marked with blue constant parameters and red variable parameters. With $L_{1}=4.0 \mathrm{~mm}, L_{2}=$ $9.27 \mathrm{~mm}, L_{3}=8.0 \mathrm{~mm}, W_{2}=0.3 \mathrm{~mm}$ and $W_{3}=1.53 \mathrm{~mm}$, the resonance frequency of the filter can be shifted easily by simply 
adjusting the parameters of the CLL resonator. For example, the S-parameters for the filtenna with $W_{l}=2.2 \mathrm{~mm}$ are given in Fig. 2(a), while those for $W_{l}=1.4 \mathrm{~mm}$ are given in Fig. 2(b). Both figures provide the simulated S-parameters for three cases: the monopole alone, the filter alone, and the filtenna. From Fig. 2(a) one finds the $-10 \mathrm{~dB}$ impedance bandwidth of the monopole antenna, the filter, and the filtenna are, respectively, $137.6 \mathrm{MHz}$ (i.e., $10.9 \%$ fractional bandwidth (FBW)), $38.5 \mathrm{MHz}$ (i.e., 3.0\% FBW), and $37.2 \mathrm{MHz}$ (i.e., 2.9\% FBW). It is obvious that the resonance of antenna and the filter were designed to coincide. The BW of the resulting filtenna is narrower than that of its monopole antenna, being limited by the filter. On the other hand, the narrow band filter endows the antenna with the desired frequency-selection characteristic. This straightforward combination of antenna and filter is routinely adopted [3], [4]. In contrast, the results in Fig. 2(b) show that the impedance resonance frequency of the monopole antenna and the filter do not coincide. Nonetheless, the resulting filtenna exhibits a wider bandwidth: $42.6 \mathrm{MHz}$ (i.e., 3.1\% FBW) even though its resonance frequency has been shifted by $\sim 100 \mathrm{MHz}$. While such a combination could lead to impedance mismatch issues, our simulation studies demonstrate that it does not while providing advantages over other choices.

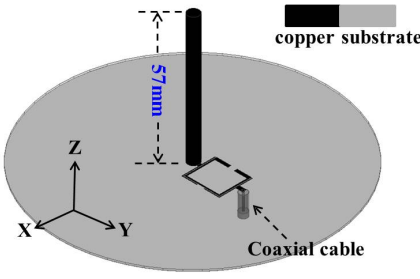

(a)

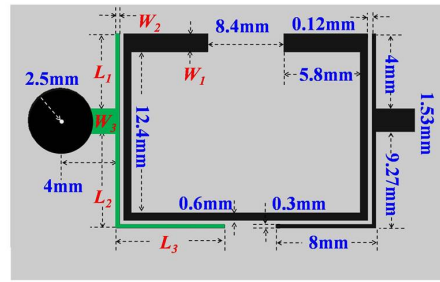

(b)
Fig. 1 Band-pass filtenna model and its design parameters. (a) 3-D isometric view. (b) Top view.

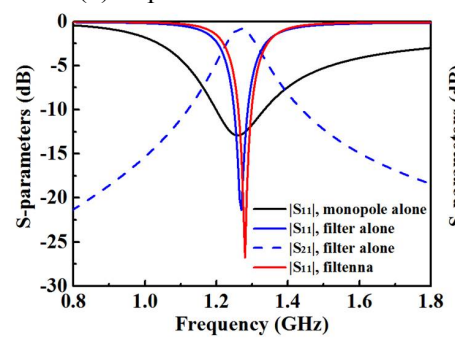

(a)



(b)
Fig. 2 The simulated S-parameters of the monopole alone, the filter alone, and their optimized combination into a filtenna. (a) $W_{l}=2.2 \mathrm{~mm}$. (b) $W_{l}=1.4 \mathrm{~mm}$.

The connection between the monopole and the filter is represented by the green L-shaped microstrip coupling segment in Fig. 1(b). This element and the gap between it and the CLL resonator control the strength of their mutual coupling. By adjusting the parameters, $L_{1}, L_{3}, W_{2}$ and $W_{3}$, the operational bandwidth variation shown in Fig. 3 is observed. Note that the total edge length in all of the cases is the same, i.e., $L_{1}+W_{3}+L_{2}=$ $14.8 \mathrm{~mm}$. As $L_{1}$ decreases from 4.0 to $0 \mathrm{~mm}, W_{2}$ decreases from 0.3 to $0.1 \mathrm{~mm}$, and $W_{3}$ increases from 1.53 to $3.0 \mathrm{~mm}$, the impedance match gradually improves while the $-10 \mathrm{~dB}$ bandwidth is broadened up to $200.4 \mathrm{MHz}$ (i.e., $16.3 \% \mathrm{FBW}$ ). When the filtenna is designed with $L_{1}=0 \mathrm{~mm}, L_{2}=11.62 \mathrm{~mm}, L_{3}$ $=10.0 \mathrm{~mm}, W_{1}=1.4 \mathrm{~mm}, W_{2}=0.1 \mathrm{~mm}$, and $W_{3}=3.0 \mathrm{~mm}$, as shown in the subfigure in Fig. 4, a more than $\sim 5.2$ times FBW improvement was obtained in comparison to the band-pass filtenna in Fig. 2(a), and a more than $\sim 49 \%$ FBW improvement in comparison to the monopole antenna alone as shown in Fig. 4. Moreover, Fig. 4 also shows that this optimized filtenna possesses a flat realized gain response within its $-10 \mathrm{~dB}$ bandwidth and has good band-edge selectivity. The radiation efficiency of the monopole alone is nearly $100 \%$ while that of the filtenna averages $\sim 91 \%$ (in the range from $88 \%$ to $96 \%$ ). Simulations confirm that this reduction of efficiency results from the relatively high current density concentrated on the compact filter structure and the associated losses in its copper components and the background substrate.

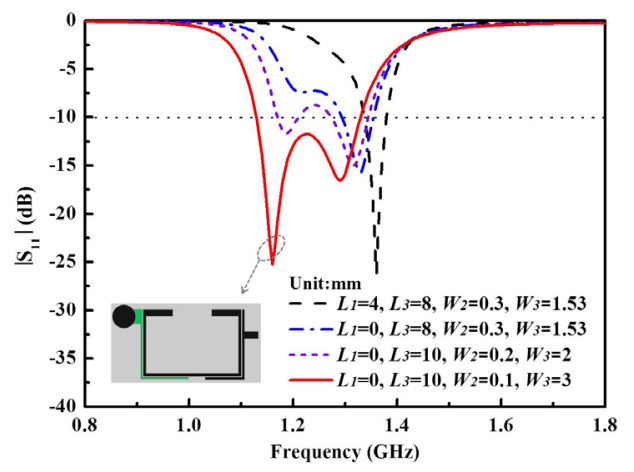

Fig. 3 Variation of the simulated $\left|\mathrm{S}_{11}\right|$ values of the modified filtenna when different $L_{1}, L_{3}, W_{2}$ and $W_{3}$ values are considered.

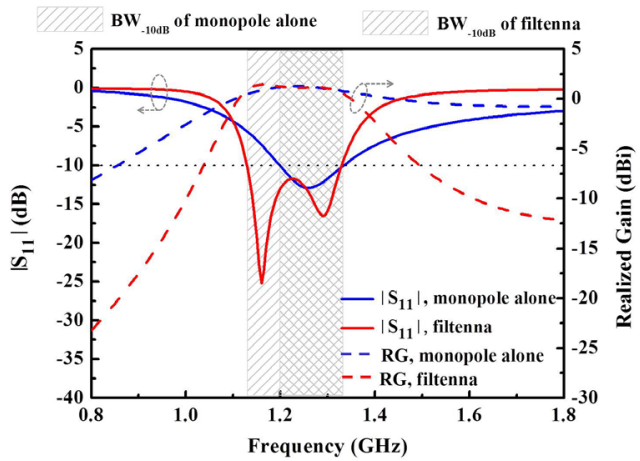

Fig. 4 The simulated $\left|S_{11}\right|$ and realized gain (RG) values of the optimized filtenna and monopole alone as functions of the source frequency.

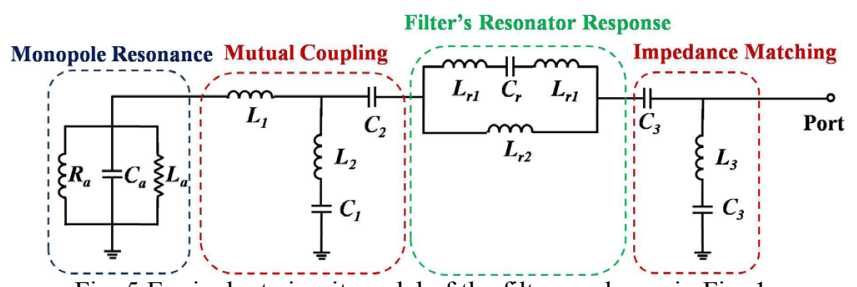

Fig. 5 Equivalent circuit model of the filtenna shown in Fig. 1.

The circuit model given in Fig. 5 helps explain the operational principles of the optimized filtenna. The input impedance of the monopole antenna near its resonance frequency is modeled as a parallel RLC resonator $\left(R_{a}, L_{a}, C_{a}\right)$ [8]. The CLL-based filter near its resonance frequency is modeled as a second LC resonator $\left(L_{r 1}, C_{r}, L_{r 1}, L_{r 2}\right)$ [9]. The green unbalanced connection line located between the antenna and the filter in Fig. 1(b) is modeled as a mutual coupling element $\left(L_{1}, L_{2}, C_{1}\right.$, and $\left.C_{2}\right)$. As illustrated with Figs. 2(b) and 3, 
when the resonance frequencies of the monopole antenna and the filter are in close proximity, but their bandwidths do not coincide, the mutual coupling level between them can be tuned by adjusting the values of $L_{1}, L_{2}, C_{1}$, and $C_{2}$. This tuning led to the increased bandwidth observed in Fig. 4. It is clear that these adjustments nicely overlapped the filter and monopole resonances to produce the observed expanded bandwidth.

\section{DESIGN OF COMPACT FILTENNAS}

The design strategy introduced in Section II can be used to obtain compact filtennas. A previously successful compact NFRP antenna was selected as the radiator [10], [11]. The filter design employed in the monopole-based filtenna example was selected for them.

\section{A. Design of a CLL-based filtenna}

The detailed filtenna configuration is depicted in Fig. 6. The compact NFRP ESA shown in Figs. 6(a) and 6(e) was selected as the radiator. Its NFRP element is printed on one side of the semicircular substrate and a monopole strip is printed on the opposite side. The combination of this radiator and the CLL resonator-based filter is shown in Figs. 6(a) - 6(d). An enlarged view of the filter is shown in Fig. 6(b). This filter structure is a typical band-pass design [7], [12], [13]. It is taken to be symmetric with respect to the S-S' line. One of its ends is connected to the printed monopole and the other is connected to the SMA.
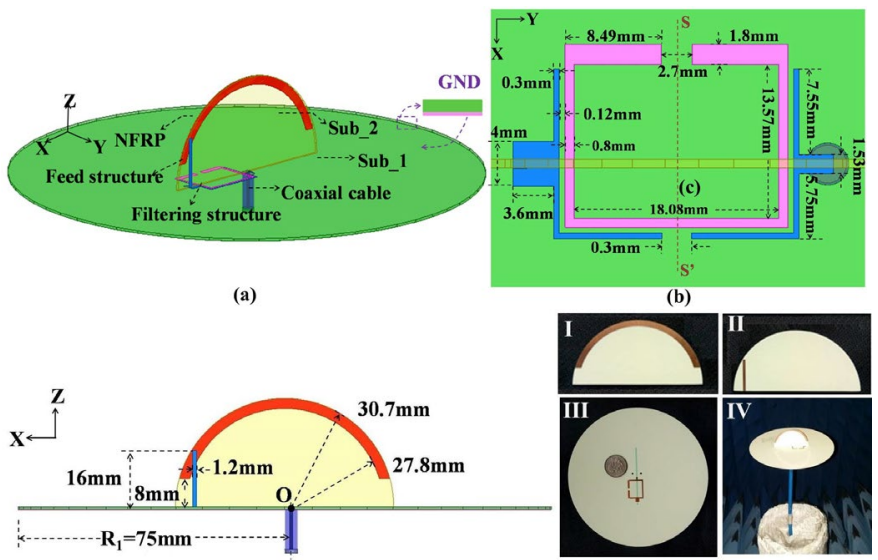

(c) (d)

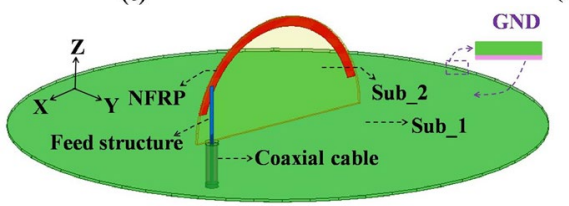

(e)

Fig. 6 The compact NFRP filtenna design. (a) 3-D isometric view of the NFRP filtenna. (b) The filter layout. (c) Side view of both the filtenna and ESA. (d) Fabricated prototype of the filtenna in different views. (e) 3-D isometric view of the NFRP ESA alone.

\section{B. Simulated and Measured Results}

The simulated and measured $\left|\mathrm{S}_{11}\right|$ and peak realized gain values as functions of the source frequency of the optimized filtenna are provided in Fig. 7. The impedance matching was quantified using an Agilent E8361A PNA vector network analyzer (VNA). The far-field radiation performance was obtained in an anechoic chamber at the National Key Laboratory of Science and Technology on Communications, University of Electronic Science and Technology of China (UESTC), which is mainly based on the Agilent N5230A PNA-L VNA and the SATIMO passive measurement system [14]. As a comparison, the simulated reflection coefficient of the optimized NFRP ESA alone (as shown in Fig. 6(e)) is included in Fig. 7. For the NFRP ESA alone, it has a $-10 \mathrm{~dB}$ impedance bandwidth of $30.3 \mathrm{MHz}$ with its center frequency being $1.26 \mathrm{GHz}$ (i.e., $2.4 \%$ FBW) with $k a_{r a d}=0.81$ (where $a_{r a d}$ is the radius of the smallest sphere that completely encloses the radiating element at its lowest operational frequency, $f_{L}$, and $k=$ $2 \pi / \lambda_{L}=2 \pi f_{L} / \mathrm{c}$ is the corresponding free space wave number). Note that the ground size is R1=75 mm, i.e, $k a_{\text {ground }} \sim 1.96$. While the ground element has no significant influence on the impedance matching and bandwidth, it impacts the gain and front-to-back ratio and, thus, cannot be ignored.

Within its operational band, the realized gain (along $+\mathrm{z}$ axis) and radiation efficiency, respectively, are in the ranges: 5.73 $5.94 \mathrm{dBi}$ and $94-95 \%$. The results for the fabricated filtenna show the predicted two overlapping resonances. Its simulated (measured) $\left|\mathrm{S}_{11}\right|_{\min }$ values are located at 1.24 (1.23) and 1.272 (1.265) GHz. The $-10 \mathrm{~dB}$ bandwidth was enhanced to 50 (49) $\mathrm{MHz}$, from 1.23 (1.223) to $1.28(1.272) \mathrm{GHz}$, i.e., the design has a $4.2 \%(3.93 \%)$ FBW. The peak realized gain values are relatively flat in this band, varying from 4.73 (4.25) to 5.43 (5.23) dBi. Compared with the simulated results of the NFRP ESA alone, the $\left|S_{11}\right|$ values near the band edges clearly confirm that this NFRP filtenna has excellent band selectivity. The realized gain curve demonstrates that the filtenna exhibits an essentially flat response over its entire operational bandwidth. It is noted that there is a transmission zero located at $0.73 \mathrm{GHz}$, but it is well below the operational band. Comparing the measured results to the simulated ones, the measured values moved slightly lower in frequency.

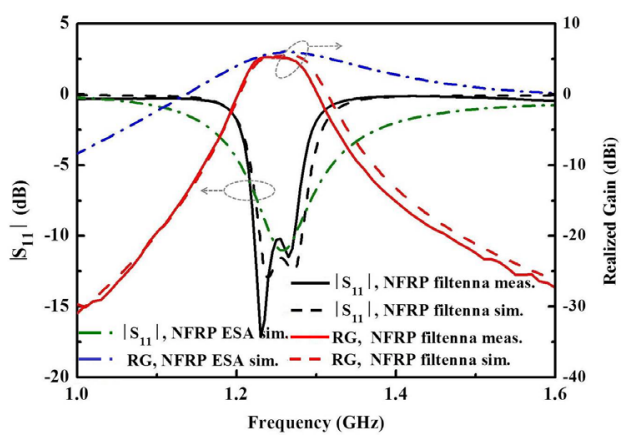

Fig. 7 The simulated and measured $\left|S_{11}\right|$ and peak realized gain values as functions of the source frequency for the filtenna design depicted in Fig. 6 and for the CLL-based NFRP ESA alone.

Fig. 8 shows the measured and simulated $E$ - and $H$-plane peak realized gain patterns of the prototype filtenna at its measured (simulated) lower resonance frequency: 1.230 (1.240) GHz. These patterns are in good agreement. The measured (simulated) peak gain was $5.12(5.36) \mathrm{dBi}$. It is also observed that the measured radiation patterns in the $E$-plane tilt a little 
away from the broadside direction. This phenomenon is ascribed to the asymmetry of the loop current amplitude generated by the CLL-based NFRP element and has been explained in detail previously [15]. The radiation pattern at the higher resonance frequency, $1.265(1.272) \mathrm{GHz}$, has the same behavior. Overall, the experimental results for the filtenna agree well with their simulated values.

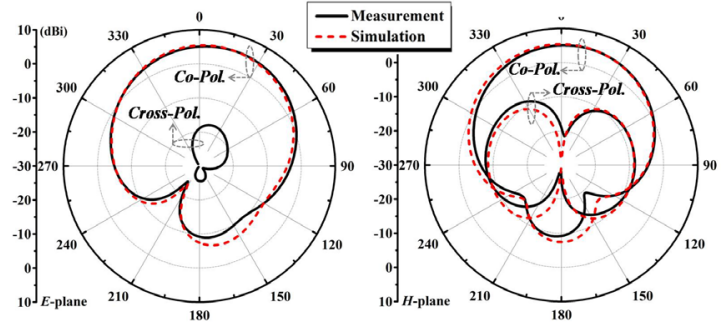

Fig. 8 The simulated (measured) peak realized gain patterns in the $E$ - and $H$-planes at the lower resonance frequency of the filtenna shown in Fig. 6: 1.24 (1.230) GHz.

\section{Current distributions}
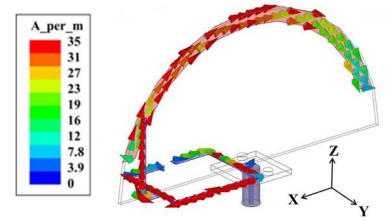

(a)

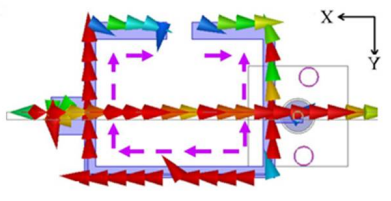

(b)
Fig. 9 Current distributions on the conductor elements of the the filtenna at its lower resonance frequency, 1.24 GHz. (a) 3-D view. (b) Top view.

The current distributions on the filtenna's conductors are shown in Fig. 9 at its lower resonance frequency, $1.24 \mathrm{GHz}$. One can see that the currents on the two strips along the y-axis in the filter structure are in-phase and are orthogonal to the current direction on the NFRP element. This behavior increases the cross-polarization level as observed in Fig. 8. It is observed in Fig. 9 that the currents are indeed strongly concentrated on the CLL resonator. As explained, this property leads to the slight decrease in the radiation efficiency value from that of the ESA alone. The current distribution behavior at the higher resonance frequency, $1.272 \mathrm{GHz}$, is essentially the same.

\section{Design of Compact Filtennas}

The design shown in Fig. 10 was varied to determine if there were any advantages to altering the configuration, orientation, and position of the filter element. Compared with the filtenna shown in Fig. 6, the NFRP element, the printed monopole pieces and the CLL resonator of the filtering part shown in Figs. 10 (a)-(c) were all left unchanged. The corresponding simulation results are presented in Fig. 11.

Fig. 11 demonstrates that all three of these filtenna designs produce two nearby resonance frequencies and, consequently, demonstrate the desired, significantly improved operational bandwidth. It also shows that there is almost no variation in the peak realized gain value along the $+\mathrm{z}$-axis across the operational band. Moreover, the enhanced selectivity at its band edges is also clearly maintained. Table I summarizes the performance characteristics of the various filtenna designs. Additionally, Table I shows that all of the simulated combinations of the radiating and filtering elements are electrically small (i.e., $k a<$ 1) and maintain about a threefold broader fractional bandwidth in comparison to the electrically small CLL-based NFRP antenna alone. Note that the presence of the filter leads in each case to a decrease in the radiation efficiency values when compared to the ESA alone.

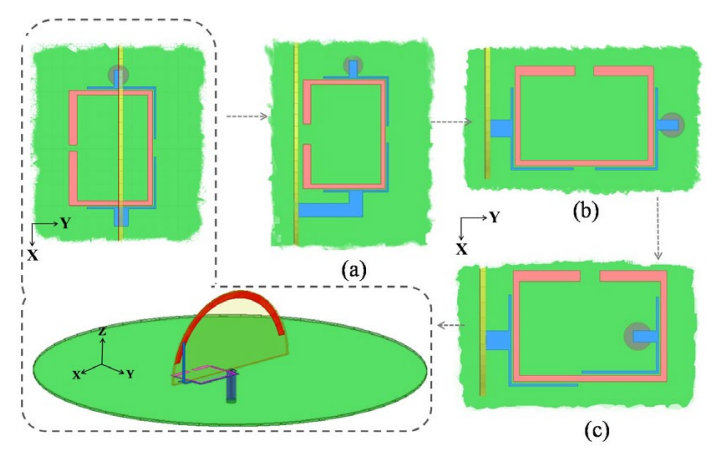

Fig. 10 Exploring variations of the filtenna design shown in Fig. 6. Change in the filter (a) position, (b) orientation, and (c) structure.

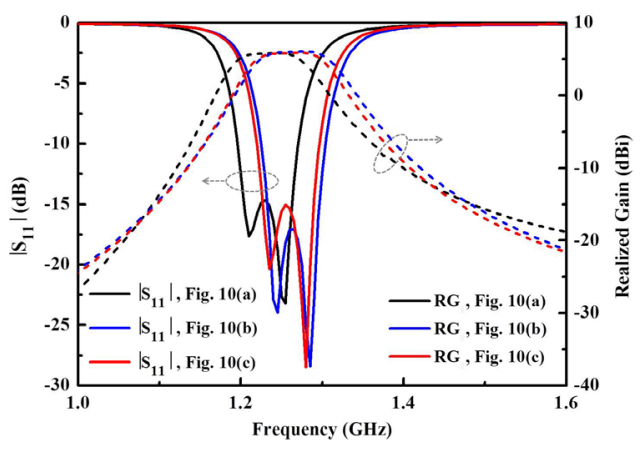

Fig. 11 The simulated $\left|S_{11}\right|$ and peak realized gain values as functions of the source frequency for the three cases shown in Fig. 10.

Table I Summary of the Performance Characteristics of the Reported Filtennas

\begin{tabular}{|c|c|c|c|c|c|}
\hline $\begin{array}{c}\text { Reported } \\
\text { filtennas }\end{array}$ & $\begin{array}{c}\text { Center } \\
\text { freq. } f_{0} \\
(\mathrm{GHz})\end{array}$ & $\begin{array}{c}\text { FBW-10dB } \\
(\%)\end{array}$ & $\begin{array}{c}k a \\
\text { Radiator } \\
\text { \& Filter }\end{array}$ & $\begin{array}{c}\text { Realized } \\
\text { Gain } \\
(\mathrm{dBi})\end{array}$ & $\begin{array}{c}\text { Radiation } \\
\text { Efficiency } \\
(\%)\end{array}$ \\
\hline Fig. 10(a) & 1.23 & 6.07 & 0.79 & $4.85-5.83$ & $76-86$ \\
\hline Fig. 10b) & 1.26 & 6.32 & 0.89 & $5.04-6.04$ & $78-88$ \\
\hline Fig. 10(c) & 1.26 & 6.13 & 0.90 & $4.92-5.89$ & $77-86$ \\
\hline ESA alone & 1.26 & 2.4 & 0.81 & $5.73-5.94$ & $94-95$ \\
\hline
\end{tabular}

\section{CONCLUSIONS}

A set of compact, bandwidth-enhanced, NFRP filtennas was demonstrated. A design strategy was introduced to enhance the impedance bandwidth of a selected electrically small, NFRP antenna by combining it with a properly tuned resonant filter. The overall filtenna design maintains stable radiation performance when the filter component is arranged in different configurations, i.e., with different relative positions and orientations. Since this design strategy leads to enhanced impedance bandwidths and to compact systems, these filtennas may prove to be useful for a variety of wireless systems. 


\section{REFERENCES}

[1] M. Barbuto, F. Trotta, F. Bilotti, and A. Toscano, "Horn antennas with integrated notch filters," IEEE Trans. Antennas Propag., vol. 63, no. 2, pp. 781-785, Feb. 2015.

[2] A. K. Horestani, Z. Shaterian, J. Naqui, F. Martín, and C. Fumeaux, "Reconfigurable and tunable $\mathrm{S}$-shaped split-ring resonators and application in band-notched UWB antennas," IEEE Trans. Antennas Propag., vol. 64, no. 9, pp. 3766-3776, Sep. 2016.

[3] G.-H. Sun, S.-W. Wong, L. Zhu, and Q.-X. Chu, "A compact printed filtering antenna with good suppression of upper harmonic band," IEEE Antennas Wirel. Propag. Lett., vol.15, pp. 1349-1352, 2016.

[4] W. Duan, X. Y. Zhang, Y.-M. Pan, J.-X. Xu, and Q. Xue, "Dual-polarized filtering antenna with high selectivity and low cross polarization," IEEE Trans. Antennas Propag., vol. 64, no. 10, pp. 4188-4196, Oct. 2016.

[5] H. Chu, C. Jin, J.-X. Chen, and Y.-X. Guo, "A 3-D millimeter-wave filtering antenna with high selectivity and low cross-polarization," IEEE Trans. Antennas Propag., vol. 63, no.5, pp. 2375-2380, May. 2015.

[6] C.-X. Mao, S. Gao, Y. Wang, Q. Luo, and Q.-X. Chu, "A shared-aperture dual-band dual-polarized filtering-antenna-array with improved frequency response," IEEE Trans. Antennas Propag., vol. 65, no. 4, pp. 1836-1844, Apr. 2017.

[7] J.-S. Hong, H. Shaman, and Y.-H. Chun, "Dual-mode microstrip open-loop resonators and filters," IEEE Trans. Microw. Theory Tech., vol. 55, no. 8, pp. 1764-1770, Aug. 2007.

[8] Y. Dong, H. Toyao, and T. Itoh, "Design and characterization of miniaturized patch antennas loaded with complementary split-ring resonators," IEEE Trans. Antennas Propag., vol. 60, no. 2, pp. 772-785, Feb. 2012.

[9] B.-F. Zong, G.-M. Wang, J.-G. Liang, and C. Zhou, "Compact bandpass filter with two tunable transmission zeros using hybrid resonators," IEEE Microw. Wireless Compon. Lett., vol. 25, no. 2, pp. 88-90, Feb. 2015.

[10] P. Jin and R. W. Ziolkowski, "Multi-frequency, linear and circular polarized, metamaterial-inspired, near-field resonant parasitic antennas," IEEE Trans. Antennas Propag., vol. 59, no. 5, pp. 1446-1459, May 2011.

[11] M.-C. Tang, T Shi, and R. W. Ziolkowski, "Electrically small, broadside radiating Huygens source antenna augmented with internal non-Foster elements to increase its bandwidth," IEEE Antennas Wirel. Propag. Lett., vol.16, pp. 712-715, 2017.

[12] J.-S. Hong and M. J. Lancaster, "Couplings of microstrip square open-loop resonators for cross-coupled planar microwave filters," IEEE Trans. Microw. Theory Tech., vol. 44, no. 12, pp. 2099-2109, Dec. 1996.

[13] X.-Y. Zhang and Q. Xue, "Novel centrally loaded resonators and their applications to bandpass filters," IEEE Trans. Microw. Theory Tech., vol. 56, no. 4, pp. 913-921, Apr. 2008.

[14] StarLab Version D User Guide 1.0, Reference: TD. 224.1.08. SATF. A, SATIMO Corporations 2008.

[15] M.-C. Tang, R. W. Ziolkowski, S. Xiao, M. Li, and J. Zhang, "Frequency-agile, efficient, near-field resonant parasitic monopole antenna," IEEE Trans. Antennas Propag., vol. 62, no. 3, pp. 1479-1483, Mar. 2014. 